\title{
Isolated hereditary diffuse palmoplantar keratoderma in Hong Kong Chinese patients: a case series
}

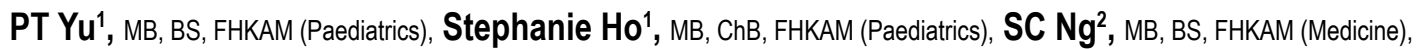
FM Lo ${ }^{1}$, MB, BS, FHKAM (Paediatrics), HM Luk ${ }^{1 *}$, MB, BS, FHKAM (Paediatrics)

${ }^{1}$ Clinical Genetic Service, Department of Health, Hong Kong

${ }^{2}$ Social Hygiene Clinic, Department of Health, Hong Kong

*Corresponding author: luksite@gmail.com

Hong Kong Med J 2021;27:358-61

https://doi.org/10.12809/hkmj208902

\section{Case reports}

\section{Patient 1}

A 26-year-old man presented with a history of hyperhidrosis of the palms and soles since age 6 months. He subsequently developed progressive palmoplantar hyperkeratosis. $\mathrm{He}$ enjoyed otherwise good past health. His parents were nonconsanguineous and there was no significant family history. Physical examination revealed bilateral symmetrical thick waxy hyperkeratosis of the palms and soles with well-demarcated transgradient margins and focal macerated erosions over the palms. Knuckle pads were observed on the dorsum of the proximal interphalangeal joints of the fingers and toes (Fig 1). There were no associated ectodermal manifestations, brachydactyly, nail abnormality or deformity. There was no insolate lesion over the elbows or knees. Medical exome sequencing identified homozygous pathogenic variants in NM_020427.2(SLURP1) c.147_150delCTGC, p.Cys $50 \%$. The molecular diagnosis of Meleda disease [MIM \#248300] was confirmed.

\section{Patient 2}

A 65-year-old woman presented with a history of diffuse hyperkeratosis of bilateral palms and soles since early infancy. She otherwise enjoyed good past health. Her parents were first-degree cousins and her younger brother had similar dermatological features. Skin biopsy revealed non-epidermolytic keratoderma. Physical examination showed diffuse yellowish to erythematous palmoplantar keratoderma (PPK) with transgradiens over bilateral palms and soles and well-demarcated margins. The fingers were mutilating with camptodactyly and cutaneous syndactyly. There was total nail dystrophy of all fingernails and toenails but no insolate lesions over the elbows or knees (Fig 1). Whole exome sequencing revealed homozygous likely pathogenic variants in SLURP1 NM_020427.2(SLURP1) c.256G >A, p.Gly86Arg. The molecular diagnosis of Meleda disease [MIM \#248300] was confirmed.

\section{Patient 3}

An 18-year-old woman presented with mild bilateral diffuse erythematous palmoplantar keratosis extending to the dorsal surfaces of the hands and feet since early infancy (Fig 1). There was no associated systemic involvement and no significant family history. Skin biopsy (Fig 2) revealed marked compact orthokeratosis, focal parakeratosis, mild hypergranulosis, acanthosis and psoriasiform epidermal hyperplasia, as well as mononuclear inflammatory infiltrate in the dermis.

Medical exome sequencing identified biallelic pathogenic variants in NM_001040147.2(SERPINB7):c.169-1G>A, p.? and c.522dup, p.Val175Cysfs*46. The molecular diagnosis of PPK, Nagashima type [MIM \#615598] was confirmed.

\section{Discussion}

Hereditary PPK is a heterogeneous group of disorders characterised by marked hyperkeratosis of the palms and soles due to a defect in cornification. It can occur in isolation or in association with other ectodermal defects or extracutaneous manifestations. Molecular studies are sometimes crucial to reach an accurate subtype classification as there are significant overlapping clinical features and heterogeneity among different types of hereditary PPK. Depending on the morphology of the lesions, PPK is classified into three major patterns: diffuse, focal (areata or striata), and punctate. Although precise figures of incidence are lacking, PPK is generally perceived to be rare. In Asia, the most frequent type of PPK is Nagashima PPK, with an estimated prevalence of 3.1 per 10000 population. ${ }^{1}$ This article summarises the clinical features and molecular findings in three Chinese individuals with isolated diffuse hereditary PPK recruited from a single centre and highlights the significance of genetic testing in reaching an accurate classification and diagnosis.

Meleda disease (also known as Mal de Meleda) has an autosomal recessive pattern of inheritance 


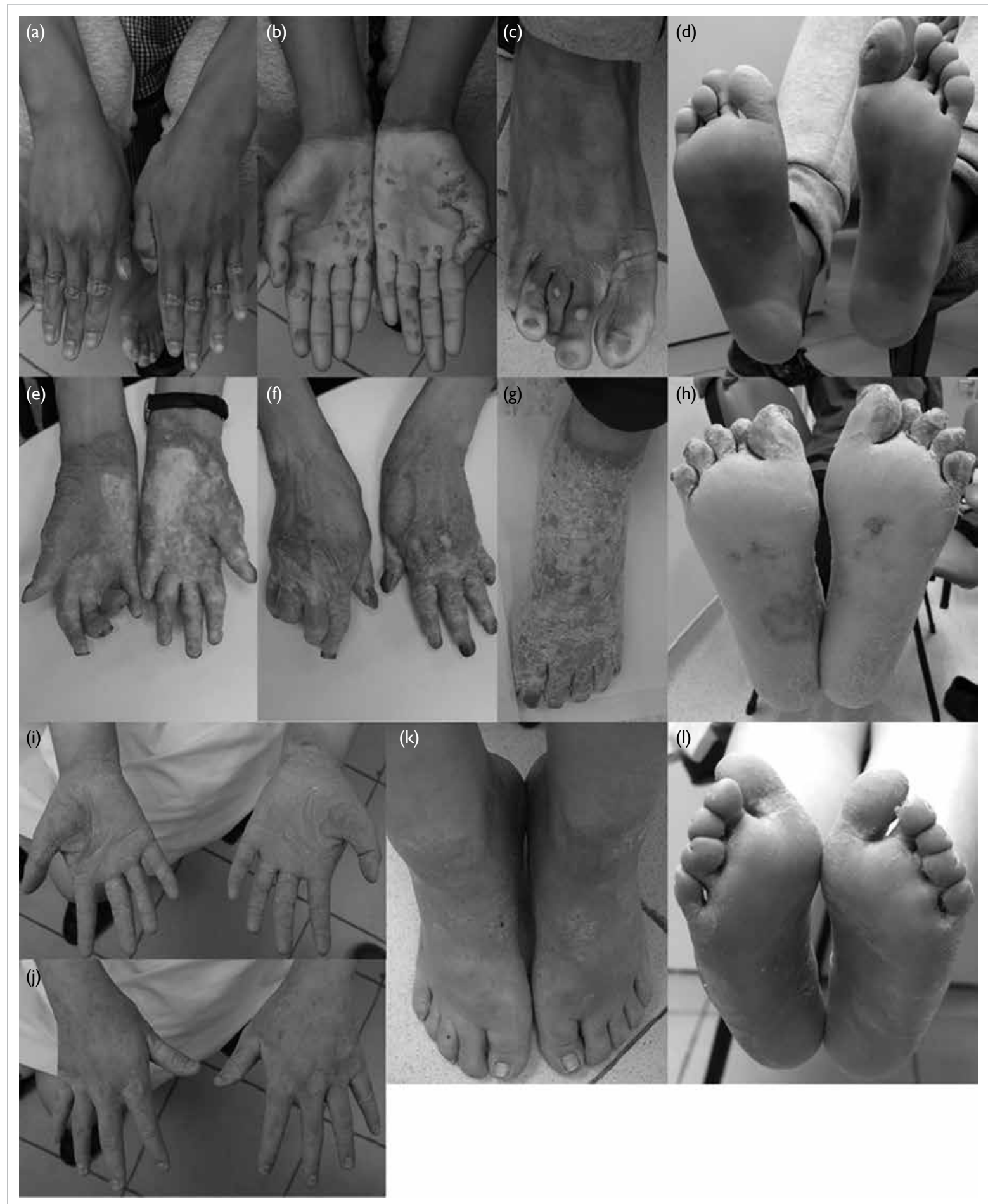

FIG I. Clinical photographs of the dermatological features of three patients with palmoplantar keratoderma (PPK). (a-d) Patient I, 26-year-old man. Bilateral thick waxy hyperkeratosis of the palms and soles with well-demarcated transgradient margins and focal macerated erosions over the palms. (b, d) Knuckle pads are evident on the dorsum of the proximal interphalangeal joints of the fingers and toes. (e-h) Patient 2 65-year-old woman. Diffuse yellowish to erythematous PPK with transgradiens over bilateral palms and soles. Tapering of the fingertips with mutilating features such as flexion contracture of the fingers and cutaneous syndactyly are evident. There is also total nail dystrophy of all nails. (i-l) Patient 3, 18-year-old woman. Clinical appearance of Nagashima-type PPK: mild bilateral diffuse erythematous palmoplantar hyperkeratosis extending to the dorsal surfaces of the hands and feet with well-demarcated borders 


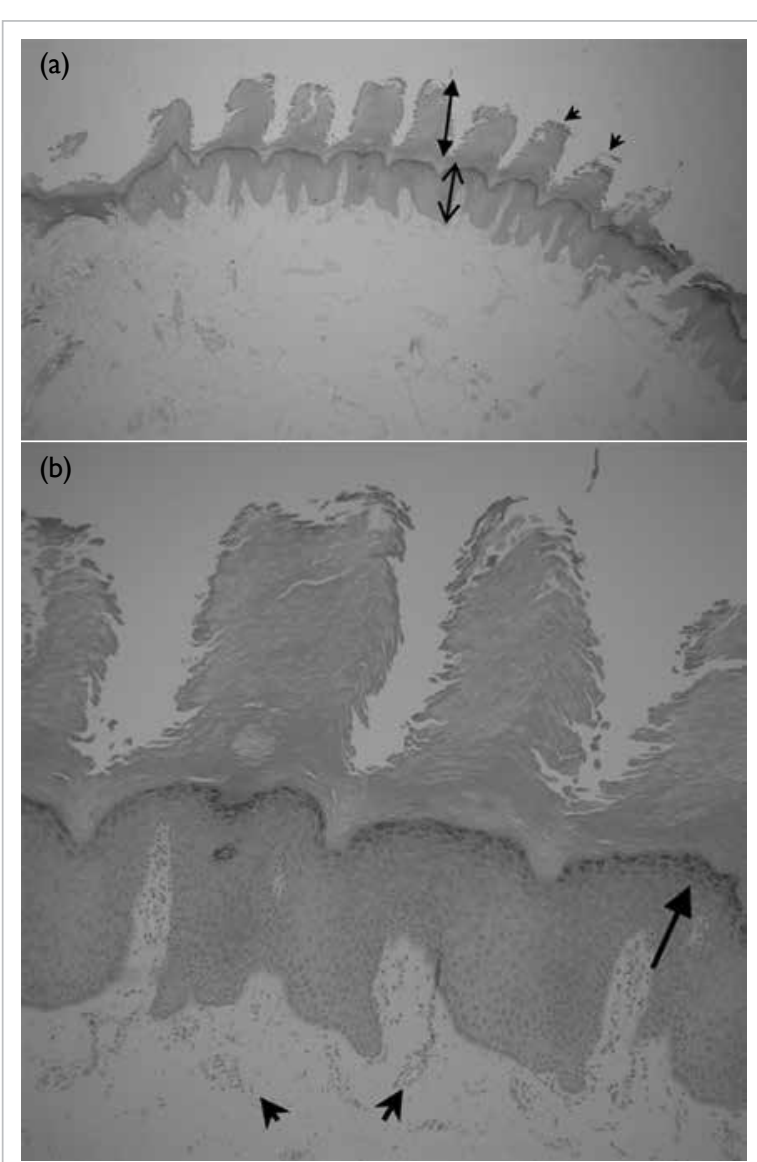

FIG 2. Histology of skin biopsy from Patient 3 showing (a) marked compact orthokeratosis (closed arrows), acanthosis and psoriasiform epidermal hyperplasia (open arrows), and focal parakeratosis (arrowheads) [haematoxylin and eosin, $\times 25]$, and (b) mild hypergranulosis (closed arrow) and mononuclear inflammatory infiltrate (arrowheads) in the dermis (haematoxylin and eosin, $\times 100$ )

and is characterised by an early onset of bilateral diffuse, well-demarcated thick and waxy PPK in a glove and stocking pattern. It is rare, with a prevalence of 1:100000. ${ }^{2}$ Palmoplantar erythema can be evident from early infancy and tends to progress to thick hyperkeratosis as an affected individual ages. Meleda disease is commonly associated with hyperhidrosis and nail anomalies such as koilonychia, onychogryphosis, and subungual hyperkeratosis. Anomalies of the digits, including pseudoainhum, contracture, tapering of digits, knuckle pads and fifth-finger dysplasia have been reported. Oral manifestations including lower-lip angular cheilitis, high arch palate and perioral erythema can be variably present. Skin biopsy is characterised by histological findings of hyperkeratosis and acanthosis in the epidermis without evidence of epidermolysis. ${ }^{2}$ SLURP-1 was subsequently identified to be responsible for Meleda disease in 2001 by
Fischer et al. ${ }^{3}$ SLURP-1 is involved in regulation of inflammation and keratinocyte apoptosis. Treatment options include oral retinoids, topical keratolytics, and surgical excision of hyperkeratosis followed by placement of a full-thickness skin graft. Infection is treated with antibacterials and antifungals, with some centres advocating the use of prophylactic topical antifungals due to a susceptibility to tinea infection.

Nagashima-type PPK (NPPK) is probably the most common form of PPK among patients of Asian ethnicity. It was initially described by Nagashima ${ }^{4}$ in 1977. Biallelic mutations of SERPINB7 were found to be causative of NPPK in 2013. ${ }^{5}$ The hyperkeratosis is non-progressive after puberty and has milder clinical features when compared with Meleda disease. It is characterised by diffuse mild erythematous nonmutilating palmoplantar hyperkeratosis that can transform into a spongy white appearance after immersion in water. The dorsal surface of the hands and feet, ankles, Achilles tendon area, elbows and knees can be involved. Palmoplantar hyperkeratosis occurs in isolation without associated ectodermal or extracutaneous manifestations. Histological findings are unremarkable with orthohyperkeratosis, acanthosis and mild perivascular inflammatory infiltration of lymphocytes in the upper dermis. ${ }^{1} \mathrm{~A}$ nonsense c.796C $>\mathrm{T}$ founder mutation in SERPINB7 has been reported to be prevalent in Chinese patients with NPPK, ${ }^{1}$ but c.169-1G $>$ A in Patient 3 is novel to the literature. No curative treatment is available but topical application of gentamicin has been investigated and appears promising. ${ }^{6}$

\section{Conclusion}

We have reported three cases of PPK that illustrate the clinical phenotype and molecular findings of this particular subgroup of genodermatoses. Molecular investigations may be warranted for accurate diagnosis of PPK and to determine its inheritance, and may be beneficial in reproductive management within the family.

\section{Author contributions}

All authors contributed equally to the concept or design of the study, acquisition of the data, analysis or interpretation of the data, drafting of the manuscript, and critical revision of the manuscript for important intellectual content. All authors had full access to the data, contributed to the study, approved the final version for publication, and take responsibility for its accuracy and integrity.

\section{Conflicts of interest}

The authors have no conflicts of interest to disclose.

\section{Acknowledgement}

The authors thank Dr WC Siu for preparing the histology photographs. 


\section{Funding/support}

This study received no specific grant from any funding agency in the public, commercial, or not-for-profit sectors.

\section{Ethics approval}

The patients were treated in accordance with the Declaration of Helsinki. The patients provided written informed consent for all treatments and procedures, and for publication of this report.

\section{References}

1. Yin J, Xu G, Wang H, et al. New and recurrent SERPINB7 mutations in seven Chinese patients with Nagashima-type palmoplantar keratosis. J Invest Dermatol 2014;134:226972.
2. Perez C, Khachemoune A. Mal de Meleda: a focused review. Am J Clin Dermatol 2016;17:63-70.

3. Fischer J, Bouadjar B, Heilig R, et al. Mutations in the gene encoding SLURP-1 in Mal de Meleda. Hum Mol Genet 2001;10;875-80.

4. Nagashima M. Palmoplantar keratosis. In: Miura O, Ochiai K, editors. Handbook of Human Genetics [in Japanese]. Vol 9. Tokyo: Igaku Shoin; 1977: 23-7.

5. Kubo A, Shiohama A, Sasaki T, et al. Mutations in SERPINB7, encoding a member of the serine protease inhibitor superfamily, cause Nagashima-type palmoplantar keratosis. Am J Hum Genet 2013;93:945-56.

6. Ohguchi Y, Nomura T, Suzuki S, et al. Gentamicin-induced readthrough and nonsense-mediated mRNA decay of SERPINB7 nonsense mutant transcripts. J Invest Dermatol 2018;138:836-43. 